\title{
Determining Production Quantity Models for Short-Age (Perishable) Products
}

\author{
Dadang Arifin", Zaenal Muttaqien, Rinto Yusriski \\ Department of Industrial Engineering, Universitas Jenderal Achmad Yani, Indonesia \\ Received February 25, 2020; Revised May 20, 2020; Accepted May 28, 2020
}

Copyright (C2020 by authors, all rights reserved. Authors agree that this article remains permanently open access under the terms of the Creative Commons Attribution License 4.0 International License

\begin{abstract}
This paper contains a decision-making model to determine production quantity for short-lived products or perishable products. The decision model is in the form of a mathematical formulation or as an algorithm. This research departs from the real problem that short-lived products have a considerable amount and are very closely related to daily life. The majority of these products are related to daily consumption such as processed food or beverage products, fruits and vegetables. The facts show that the number of companies engaged in this field is very large, ranging from large-scale companies, medium scale and small scale. Therefore this decision-making model is important for companies, in order to be able to improve their performance by making an appropriate or optimal production strategy. The unoptimal production levels will cause problems such as shortages, overstock which results an expired products, and wasteful costs. Departs from the real problem, then conducting a literature study to find out whether the model to be developed had been developed by previous researchers. The researcher looks for a model that is relevant to this case, at least getting a model that has similarities to be used as an additional reference. The author hopes that, this model will provide practical benefits for practitioners and academics, especially students.
\end{abstract}

Keywords Optimal, Stockout, Overstock, Expired, Costs

\section{Introduction Section}

In 2018 the food and beverage industry will become a priority subsector as a driver for achieving the 2018 non-oil and gas industry growth target of 5.67 percent (TEMPO.CO. Jakarta). The food and beverage industry sector in Indonesia also contributes $34 \%$ to the Indonesian industry, "said Minister of Industry, February 8, 2018. With the continued development of technology resulting in increasingly intense business competition, not a few industries are closed due to the impact. However, he believes that the food and beverage industry will continue to be an industry that continues to be needed by the market. "We still need food that goes into the stomach. We can't have virtual eating," he said.

The majority of food and beverage products generally have a relatively short life (non-durable goods), when compared to other products. This requires food and beverage entrepreneurs to be able to make the best strategy so that the products produced sell well in the market and do not experience expiration. While the types of products with short-age are relatively more in number compared to products with long life (durable goods). This is dominated by daily food products such as cakes, drinks, vegetables and fruit that are needed throughout human life.

The level of demand for these items always an increasing trend along with the growth of human populations and other living things. This provides an opportunity and challenge for entrepreneurs in this field to be able to manage the product as well as possible. One of the things that must be considered in managing the business is determining the best production strategy by paying attention to aspects of cost, aspects of technology and capacity, as well as the age of the product.

Some research on dealing with short-lived (perishable products) has been done quite a lot, some of them are, Jing Chen et al., (2018) developed a model to consolidate the delivery of perishable products by considering the freshness level. Liu Hengyu et al., (2018) developed a model for determining the optimal level of purchase and a policy of replenishing supplies for seasonal agricultural products. Zhixiang Chen et al., (2018) Developing production and inventory optimization models by controlling prices and promotional efforts for perishable products in the case of single suppliers and multi-buyers. Linh N.K. Duong et al., (2015) developed a multi-criteria inventory management system for substitute and perishable products. Arifin Dadang et al., (2019) 
depeloved material procurement model multi item single supplier considering life time and minimum order quantity.

Based on the above reference, the researcher considers that it is necessary to develop a model to determine the optimal level of production for short-lived products to minimize shortages and expiration. Therefore, this paper contains the development of a model with a title like the above.

One of the companies sampled in this study was a meatball maker company located in Bandung district, on Purwakarta street, Padalarang District. The company makes continuous production at very high production rates to a certain level of production (batch). With the large variety of products made through several of the same facilities the policy is considered more appropriate. To anticipate fluctuated demands, the company produces continuously by always making more than planned. With this policy, factories often get excess (over production). While meatball products have a relatively short age of around 2 days, although they can still be stored in the refrigerator. For starters, in this paper the development of the model starts from the simple one, which is the deterministic case of a single item and then continues to be a more complex, probabilistic, and multi-item model. On that basis the authors develop a model to determine the optimal level of production that is able to minimize deficiencies, minimize expired products, and minimize the total costs involved.

\section{Model Development}

The development of the model departs from the basic model developed in the material management book, Ricard J Tersine and Edward A. Silver et al., (1998). Figure 1 below show illustration of the real system.

The new model was developed by considering the age of the product, so the problem can be illustrated in the graphic illustration in Figure 2. The model was developed with the following construction:

1. Production capacity is indicated by the rate of production per day is $\mathrm{p}$

2. The level of need indicated by the rate of demand per day is $r$

3. The strategy that will not be achieved is to determine the level of production (batch) of the production cycle

4. Collection or removal of finished goods from storage is assumed to be random or based on the order of completion (first in first out).

5. Items that have expired are considered losses that cannot be processed.

The problem can be described by figure 2 with mathematical formulation below.

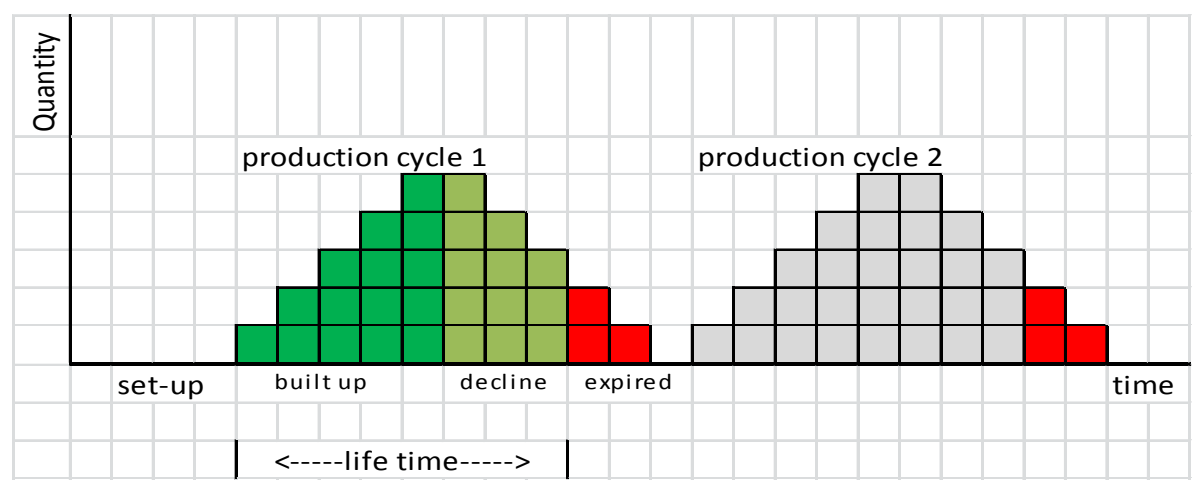

Figure 1. Illustration of the real system 


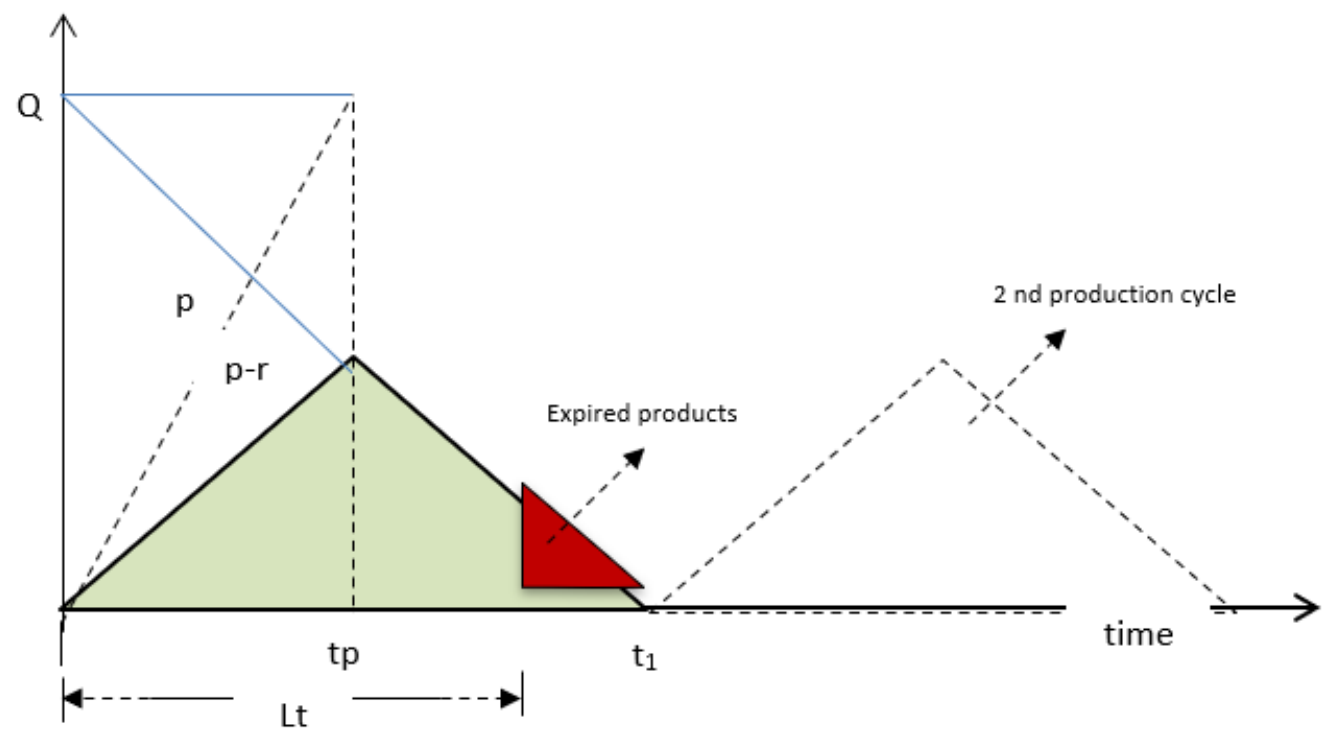

Figure 2. Model illustrated stock position

Where:

tp $=$ time of production period in one production cycle

$\mathrm{Lt}=$ product age or life time

$\mathrm{p}=$ production rate per unit time

$\mathrm{r}=$ demand rate per unit time

$\mathrm{Q}=$ production quantity in each production cycle

There are three objectives to be achieved in the development of this model, first is to determine the size of production Q so that no expired products occur with the assumption of random goods taking, and the second is to determine the amount of production $\mathrm{Q}$ if the goods are taken based on the order of completion or FIFO (first in first out). Then, determine the optimal Q for both of these assumptions.

Case 1

The first case is to determine the size of a production (batch) to avoid the occurrence of expired products with the assumption that the product is taken randomly. It is expected that the product will not expired so this can be written like this

$$
t_{1} \leq L t
$$

Where $\mathrm{t} 1$ consists of inventory built up time (tp) plus inventory depletion time (start from tp to $\mathrm{t} 1)$ or $(\mathrm{t} 1-\mathrm{tp})$, then $\mathrm{t} 1$ can be written

$$
t_{1}=t p+\frac{(p-r) t p}{r} \text { and then } L t \geq t p+\frac{(p-r) t p}{r}
$$

Then elaborated

$$
\begin{aligned}
& t p+\frac{(p-r) t p}{r}=\frac{Q}{p}+\frac{(p-r) t p}{r} \leq L t \\
& \frac{Q r+(p-r) Q}{p r} \leq L t \\
& p Q \leq L t . p . r \\
& Q \leq L t . r
\end{aligned}
$$

Therefore the product will expire if $Q>L t . r$ so the number of products that expire in each cycle is a $(Q-L t . r)$ unit. The number of products that expire in one year is $(Q-L t . r) \frac{R}{Q}$, where $\mathrm{R}$ is the annual requirement. If the cost or loss due to expiration is $\mathrm{E}$, then the annual total cost by considering the expiration cost is

$$
\begin{aligned}
T C(Q) & =P R+C \frac{R}{Q}+\frac{H Q(p-r)}{2 p}+(Q-\text { Lt.r }) \frac{R}{Q} \cdot E \\
& =P R+C \frac{R}{Q}+\frac{H Q(p-r)}{2 p}+R E-\frac{R L t . r . E}{Q}
\end{aligned}
$$

The total annual cost is a function of the level of production of $\mathrm{Q}$, therefore to get $\mathrm{Q}$ which minimizes the total cost is done by making the equation above for $\mathrm{Q}$ and is set to zero. So the size of production that minimizes total costs can be calculated by,

$$
Q=\sqrt{\frac{2 p R(C-\text { Lt.r.E })}{H(p-r)}} \text {, when } C<\text { Lt.r.E the formulation }
$$

becomes unrealistic so it is necessary to use an absolute sign $\mid(C-$ Lt.r.E $) \mid$, so that

$$
Q=\sqrt{\frac{2 p R \mid(C-\text { Lt.r.E }) \mid}{H(p-r)}}
$$




\section{Second derivative test}

$$
\frac{d^{2} T C(Q)}{d^{2} Q}=2 \frac{R}{Q^{3}}(C-\text { Lt.r.E })
$$

When $\mid(C-$ Lt.r.E $) \mid$ used so that the second derivative value will always be positive, so the $\mathrm{Q}$ value with the formula above produces the minimum value.

Case 2

If the collection is carried out in the order of work (FIFO)

In the zero time period until tp there will be an increase in inventory by $(\mathrm{p}-\mathrm{r})$ units per day, and shipping will occur for $r$ per day. If $L t>1$ day and if $(p-r)<r$ or $p<2$, the period will not expire.

In the period tp to $t_{1}$ is called inventory depletion time. If the inventory depletion time is smaller than $\mathrm{Lt}$, it will not expire.

$$
\begin{aligned}
& \frac{(p-r) t p}{r} \leq L t \\
& \frac{(p-r) Q}{p r} \leq L t
\end{aligned}
$$

So that $\mathrm{Q}$ is calculated by

$$
Q \leq \frac{L t \cdot p \cdot r}{(p-r)}
$$

\section{Implementation and Discussion}

At this stage an example of the use of the developed model will be presented followed by validation. Given the limit on the number of pages, the models that will be tried are the first 2 formulas, while the optimization model will be presented in the next article.

In the company, the research produces as many as 10 types of meatballs which are grouped into 3 categories, namely small size meatballs (one type), medium (4 types), and large size (5 types). For an example of using this single item model, large size meatballs are used. It is known that the average demand for large size meatballs is 1482 units per day. With a production speed of 1.02 seconds per unit, production capacity or speed per day in an average shift is 4940 units per day. If the meatballs have a maximum age of 2 days, then the production size of each batch is a $Q \leq L t * r=2 * 1482=2964$ unit. With that size, it will not expire, if it is assumed that the taking is done randomly.

But if the collection is carried out in the order of production or adheres to the system (first in first out), the maximum production size is calculated using the following formula,

$$
Q \leq \frac{\text { Lt.p.r }}{(p-r)}=\frac{2 * 4940 * 1482}{3458}=4234
$$

This means that if it is produced using a maximum capacity with a batch size of 4234 units, it will not expire. The size only takes 0.857 days or about 6.0 hours. The maximum inventory level will be reached at 2964 units, and will be exhausted within the next 2 days so that no product has expired. Validation of this calculation can be seen in the table below. The calculation time period is converted into units of hours, and the processing time per batch of is rounded to 6 hours. The number of effective working hours is 7 hours per day.

The results of the validation in table 1, show no difference in batch size between the calculation of the formula and the calculation of the table. Nevertheless the formulation above is still valid. One cycle of production is carried out for about 3 workdays, namely the first day for 6.0 hours for production activities, then rest for the next 2 days to spend inventory. It was during this pause that it was used to produce other products.

Table 1. Production Rate, demand Rate and Stock position

\begin{tabular}{ccccc}
\hline Hour & $\begin{array}{c}\text { Production } \\
\text { Cumulatif }\end{array}$ & $\begin{array}{c}\text { variable } \\
\text { demand }\end{array}$ & $\begin{array}{c}\text { Cumulatif } \\
\text { Demand }\end{array}$ & $\begin{array}{c}\text { Inventory } \\
\text { built-up/dec } \\
\text { line }\end{array}$ \\
\hline 1 & 706 & 0 & 0 & 706 \\
2 & 1,411 & 450 & 450 & 961 \\
3 & 2,117 & 0 & 450 & 1667 \\
4 & 2,823 & 500 & 950 & 1873 \\
5 & 3,529 & 0 & 950 & 2579 \\
6 & 4,234 & 475 & 1425 & 2809 \\
7 & & 0 & 1425 & 2809 \\
8 & & 0 & 1425 & 2809 \\
9 & & 495 & 1920 & 2314 \\
10 & & 0 & 1920 & 2314 \\
11 & & 0 & 1920 & 2314 \\
12 & & 700 & 2620 & 1614 \\
13 & & 0 & 2620 & 1614 \\
14 & & 400 & 3020 & 1214 \\
15 & & 0 & 3020 & 1214 \\
16 & & 0 & 3020 & 1214 \\
17 & & 000 & 3620 & 614 \\
18 & & 0 & 3620 & 614 \\
19 & & 0 & 3620 & 614 \\
20 & & 0 & 14 \\
\hline
\end{tabular}

The calculation results show that the maximum production size that does not cause the product to expire is 4234 units which will be finished working for 6.0 hours. After the production period there is a gap of 2 days to spend the remaining stock. 


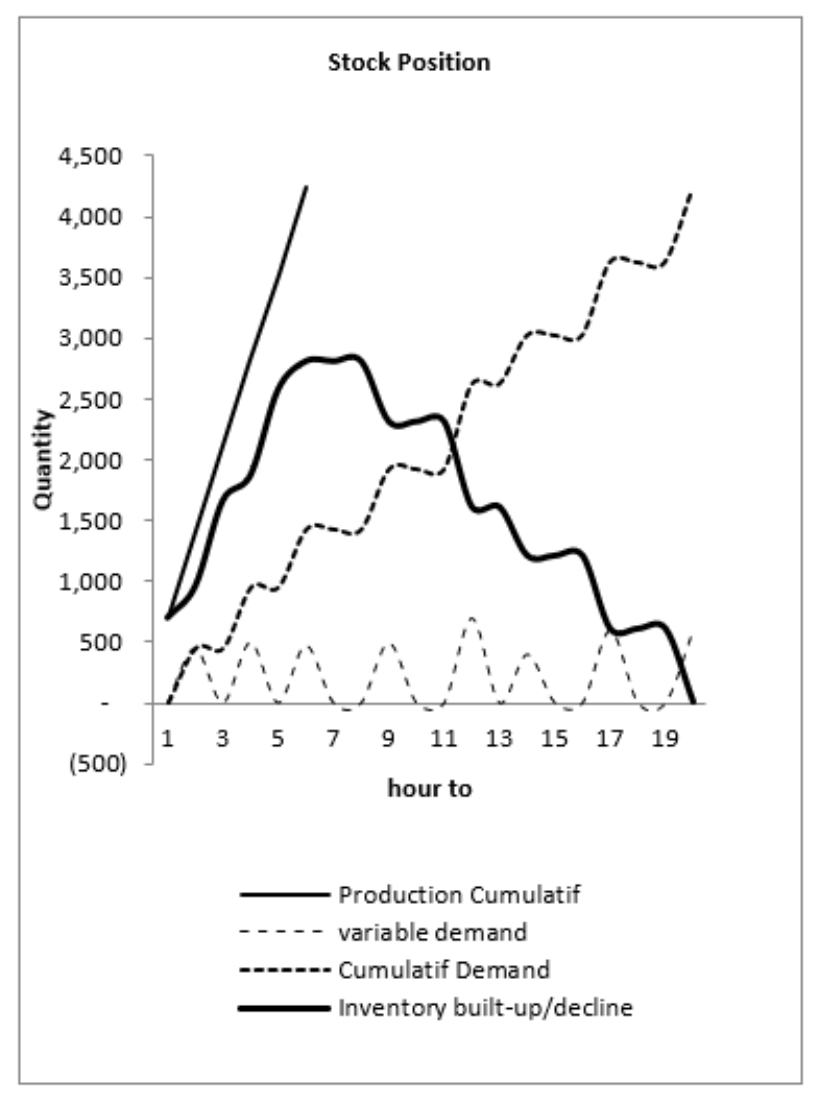

Figure 3. Stock Position

\section{Conclusions and Suggestions}

From the developed model, several conclusions can be obtained, as followed

1. The developed model is able to calculate the maximum production level to avoid expired products.

2. The developed Q batch size is not yet an optimal size, but only as a feasible initial size to avoid expiration.

3. Batch size is strongly influenced by production rate, demand rate, and product age or age.

4. The model is developed based on the assumption that the rate of production, the rate of demand, and related costs are considered certain.

\section{REFERENCES}

[1] Benjamin Blanchard, Logistics Engineering and Management, sixth edition, 2004 Prentice-Hall.

[2] Donald J Bower Sox, Manajemen Logistik, Sinar Grafika Offset, 2002

[3] Edward A. Silver, David F. Pyke, Rein Peterson, Inventory Management and Production Planning and Scheduling, Third edition, 1998 John Wiley \& Son Inc.

[4] Jing Chen ${ }^{\mathrm{a}}$ Ming Dong ${ }^{\mathrm{b}}$ Lei $\mathrm{Xu}^{\mathrm{c}}$ A perishable product shipment consolidation model considering freshness-keeping effort, Transportation Research Part E: Logistics and Transportation Review, Volume 115, July 2018, Pages 56-86, ScinceDirect, ELSEVIER.

[5] Liu Hengyu, Zhang Juliang, Zhou Chen, RuYihong, Optimal purchase and inventory retrieval policies for perishable seasonal agricultural products, Omega Volume 79, September 2018, Pages 133-145, ScinceDirect, ELSEVIER.

[6] Zhixiang Chen, Optimization of production inventory with pricing and promotion effort for a single-vendor multi-buyer system of perishable products, International Journal of Production Economics, Volume 203, September 2018, Pages 333-349, ScinceDirect, ELSEVIER.

[7] Linh N.K. Duong, Lincoln C. Wood, William Y.C. Wang., $A$ multi-criteria of inventory management system for perishable and substitutable products', ScienceDirect, ELSEVIER, 2015.

[8] Richard J Tersine, Economic Production Quantity, Material Management and Inventory Analysis, Texbook

[9] Arifin Dadang, Yusuf Eddy, Charisma Cantri, Fixed Order Quantity Model for Multi Item Single Supplier Considering Life Time and Minimum Order Quantity, Journal of Physics: Conference Series, Volume1, 179, 2019 - IOPscience, iopscience.iop.org 